\title{
Editorial \\ Breast cancer stem cell markers - the rocky road to clinical applications
}

\author{
Gabriela Dontu
}

Department of Internal Medicine, University of Michigan, Ann Arbor, Michigan 48109, USA

Corresponding author: Gabriela Dontu, gdontu@med.umich.edu

Published: 15 September 2008

Breast Cancer Research 2008, 10:110 (doi:10.1186/bcr2130)

This article is online at http://breast-cancer-research.com/content/10/5/110

(c) 2008 BioMed Central Ltd

See related research article by Honeth et al., http://breast-cancer-research.com/content/10/3/R53

\begin{abstract}
Lately, understanding the role of cancer stem cells in tumor initiation and progression became a major focus in stem cell biology and in cancer research. Considerable efforts, such as the recent studies by Honeth and colleagues, published in the June issue of Breast Cancer Research, are directed towards developing clinical applications of the cancer stem cell concepts. This work shows that the previously described CD44+CD24- stem cell phenotype is associated with basal-type breast cancers in human patients, in particular BRCA1 inherited cancers, but does not correlate with clinical outcome. These very interesting findings caution that the success of our efforts in translating cancer stem cell research into clinical practice depends on how thorough and rigorous we are at characterizing these cells.
\end{abstract}

The cancer stem cell model, a concept initially proposed more than a century ago, has been revisited with increasing enthusiasm in the past decade. This model proposes that tumors, like normal tissues, are organized in a cellular hierarchy, in which 'cancer stem cells' are the only cells with unlimited proliferation potential and therefore capable of driving tumor growth and metastasis. The 'differentiated' cancer cells that account for the majority of the tumor population may have high proliferation potential, but it is not unlimited. It follows that eliminating these differentiated progenies while sparing the cancer stem cells will ultimately result in relapse. The other component of the stem cell model of carcinogenesis holds that stem and progenitor cells are the cells susceptible to transformation, owing to their long life and high proliferative capacity.

A paradigm-shifting hypothesis, the cancer stem cell model could potentially be the foundation for new preventive and therapeutic strategies in cancer. However, concerns regarding the validity of this model have been expressed, mostly regarding its experimental validation. It has been brought to attention that xenografting cancer cells in immunosupressed animals, the gold standard for testing cancer stem cell properties in vivo, may select for cells adaptable to the animal host, therefore introducing an unavoidable bias. Experts in the field cautioned against oversimplified views that do not take into account the genetic variability and clonal evolution of cancer cells, including those of cancer stem cells.

The efforts of numerous recent studies focused on testing the validity and universality of this model across tumor types of various tissues, and on exploring its clinical implications. In line with these directions, the recent study by Honeth and colleagues [1] aims to identify possible correlations between the representation of tumor-initiating cells and classic molecular and histoclinical parameters that classify breast cancer in several distinct subtypes: basal, luminal A and B, HER2 positive and normal-like. Associations between presence of cancer stem cells in clinical samples, aggressive tumor behavior and poor clinical outcome have been speculated upon, but so far only one study shows such an association in breast cancer patients [2]. Honeth and colleagues [1] used the CD44+ CD24- phenotype to identify breast tumor initiating cells. This tumor initiating phenotype was proposed by Clarke and colleagues [3], who provided the first proof of principle for the existence of cancer stem cells in solid tumors. Their study showed that in nine breast cancer samples, a minority of cells bearing the surface markers CD44+CD24- (negatively selected for eight lineage markers in order to eliminate non-epithelial cells), were capable of generating tumors in NOD/scid mice even when implanted in low numbers. By contrast, the other cancer cell populations, such as CD44+CD24+, failed to generate tumors even when implanted in high numbers.

This ground breaking work had tremendous impact in both the stem cell field and in cancer research. At this point, it is of paramount importance to investigate the universality of these new concepts. Given the small number of tumors analyzed in the study by Clarke and colleagues [3] and the character- 
istics of these tumors (eight out of nine were pleural effusions), it is critical to inquire if the findings apply to a larger number of tumors that cover the diversity of breast cancer subtypes. Whereas the study from Hegardt's group [1] is correlative in nature, the use of a large collection of well characterized breast cancers gives weight to their conclusions. Overall, only $31 \%$ of the tumors analyzed contained CD44+CD24- cells, ranging from $1 \%$ or less to $100 \%$ of the total tumor population. The CD44+CD24phenotype was more common in basal-like tumors, and strongly associated with BRCA1 hereditary breast cancers. Given the large representation of CD44+CD24- cells in the basal layer of normal breast epithelium, these findings suggest that the cellular origin of the basal breast cancers may be a progenitor of the basal lineage. This is also consistent with the recently proposed role of BRCA1 in luminal differentiation [4]. Alternatively, or in addition, a block in luminal differentiation may lead to the same basal-like molecular phenotype, which often contains CD44+CD24cells. However, not all basal breast tumors and very few HER2 positive tumors had CD44+CD24- cells. Consistent with previous work from two other groups, the presence of CD44+CD24- cells in breast tumors did not correlate with clinical outcome $[5,6]$. No association between the CD44+/CD24- status and markers known to be important for the clinical outcome, such as tumor size, lymph node status or S-phase fraction was observed.

The findings of Honeth and colleagues raise several important questions. Some pertain to the universality of the cancer stem cell phenotype. Is the CD44+CD24- phenotype associated with tumor initiating cells only in certain breast cancers, predominantly basal-like or BRCA1? Are cancers that do not contain cells with this phenotype driven by a different cancer stem cell? If this is the case, do these cancer stem cells not bearing the CD44+CD24- phenotype have a different origin? One could speculate that they are derived from luminal progenitor cells that, upon transformation, give rise to breast cancers with these predominant molecular traits.

Other markers, such as the ALDH1 breast cancer stem cell marker, which is also present in only about 30\% of tumors, can further divide the CD44+CD24-lin- cell population into fractions that are highly tumorigenic: ALDH+CD44+CD24lin-, which is capable of generating tumors from 20 cells; and ALDH-CD44+CD24-lin-, which are not tumorigenic. Unlike CD44+CD24-lin-, the ALDH1 phenotype correlates with clinical outcome and certain histoclinical characteristics, such as tumor grade, HER2 positivity, and Ki67 status, although it is not associated with a particular molecular subtype of breast cancer [2] .

Other questions that can be raised pertain to the cancer stem cell model itself. Is it possible that the CD44+CD24phenotype is a dynamic one, and can it be lost and re- acquired during tumor progression, as a result of genetic instability and epigenetic changes? The initial findings of Clarke and colleagues indicate that this may not be the case, since in as many as four consecutive passages the hierarchy defined by the CD44+ CD24-lin- phenotype was maintained. However, recent studies show that the CD44+CD24phenotype can be induced by genetic events, such as Twist overexpression, or epigenetic events, such as exposure to transforming growth factor beta, a factor secreted by tumor associated stroma [7]. Moreover, whereas the initial work from Clarke's group described CD44 as a marker of stem cells, subsequent studies from other groups demonstrated that CD44 is functionally involved in both tumor initiation and tumor progression [7].

As Honeth and collegues conclude [1], there is much to be uncovered about cancer stem cells and their role in carcinogenesis and one should not yet exclude the coexistence of clones of independent origin and evolution, within the entire tumor population or within the cancer stem cell fraction. Whereas the stochastic model of carcinogenesis and the cancer stem cell model are exclusive, it is clear that clonal evolution contributes to the heterogeneity of cancers, even if they are hierarchically organized. Regardless of these conceptual considerations, developing clinical applications based on cancer stem cell concepts is still an ongoing effort that, despite numerous challenges, has tremendous promise.

\section{Competing interests}

The author declares that they have no competing interests.

\section{References}

1. Honeth G, Bendahl PO, Ringnér M, Saal LH, Gruvberger-Saal SK, Lövgren K, Grabau D, Fernö M, Borg A, Hegardt C: The CD44+/CD24- phenotype is enriched in basal-like breast tumors. Breast Cancer Res 2008, 10:R53.

2. Ginestier C, Hur MH, Charafe-Jauffret E, Monville F, Dutcher J, Brown M, Jacquemier J, Viens P, Kleer CG, Liu S, Schott A, Hayes D, Birnbaum D, Wicha MS, Dontu G: ALDH1 is a marker of normal and malignant human mammary stem cells and a predictor of poor clinical outcome. Cell Stem Cell 2007, 1:555-567.

3. Al-Hajj M, Wicha MS, Benito-Hernandez A, Morrison SJ, Clarke MF: Prospective identification of tumorigenic breast cancer cells. Proc Natl Acad Sci USA 2003, 100:3983-3988.

4. Liu S, Ginestier C, Charafe-Jauffret E, Foco H, Kleer CG, Merajver SD, Dontu G, Wicha MS: BRCA1 regulates human mammary stem/progenitor cell fate. Proc Natl Acad Sci USA 2008, 105:1680-1685

5. Abraham BK, Fritz $\mathrm{P}, \mathrm{McClellan} \mathrm{M}$, Hauptvogel $\mathrm{P}$, Athelogou $\mathrm{M}$, Brauch H: Prevalence of CD44+/CD24-/low cells in breast cancer may not be associated with clinical outcome but may favor distant metastasis. Clin Cancer Res 2005, 11:1154-1159.

6. Shipitsin M, Campbell LL, Argani P, Weremowicz S, BloushtainQimron N, Yao J, Nikolskaya T, Serebryiskaya T, Beroukhim R, Hu M, Halushka MK, Sukumar S, Parker LM, Anderson KS, Harris LN, Garber JE, Richardson AL, Schnitt SJ, Nikolsky Y, Gelman RS, Polyak K: Molecular definition of breast tumor heterogeneity. Cancer Cell 2007, 11:259-273.

7. Mani SA, Guo W, Liao MJ, Eaton EN, Ayyanan A, Zhou AY, Brooks M, Reinhard F, Zhang CC, Shipitsin M, Campbell LL, Polyak K, Brisken C, Yang J, Weinberg RA: The epithelial-mesenchymal transition generates cells with properties of stem cells. Cell 2008, 133:704-715. 